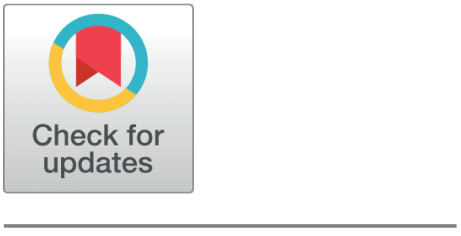

OPEN ACCESS

Received: 01.09.2020

Accepted: 20.12.2020

Published: 15.01.2021

Citation: Selasteen FD, Raj SAC (2021) A study on non-linear optical properties of Copper Sodium Tartrate single crystal for non-linear optical applications . Indian Journal of Science and Technology 14(2):

101-112. https://doi.org/

10.17485/IJST/v14i2.1561

* Corresponding author.

Tel: +919994354899

daisy.ph@bhc.edu.in

Funding: None

Competing Interests: None

Copyright: (c) 2021 Selasteen \& Raj. This is an open access article distributed under the terms of the Creative Commons Attribution License, which permits unrestricted use, distribution, and reproduction in any medium, provided the original author and source are credited.

Published By Indian Society for Education and Environment (iSee)

ISSN

Print: 0974-6846

Electronic: 0974-5645

\section{A study on non-linear optical properties of Copper Sodium Tartrate single crystal for non-linear optical applications}

\author{
F Daisy Selasteen ${ }^{1 *}$, S Alfred Cecil Raj ${ }^{2}$ \\ 1 Assistant Professor of Physics, Department of Physics, Bishop Heber College,Affiliated To \\ Bharathidasan University, Tiruchirappalli, 620 017, Tamil Nadu, India. Tel.: +91 9994354899 \\ 2 Associate Professor of Physics, School of Physical Sciences, St, Joseph's College, Affiliated To \\ Bharathidasan University, Tiruchirappalli, 620002, Tamil Nadu, India
}

\section{Abstract}

Objectives: To calculate the optical and structural parameters of the copper sodium tartrate (CUNaT) crystal for investigating its usefulness in the non-linear optics, it was analyzed by XRD and optical studies. Methods : A good nonlinear optical CuNaT crystal was grown by silica gel medium. The fundamental factors to prepare the silica gel for the controlled and defect - free growth of the CuNaT crystal are gel $\mathrm{pH}$, gel density and gel setting period. Findings:The non-centro symmetric structure with refined lattice parameters: $a=7.7230 \AA$, $\mathrm{b}=20.2964 \AA, \mathrm{c}=7.9896 \AA$ and the required functional groups: $\mathrm{O}-\mathrm{H}, \mathrm{M}-\mathrm{O}$, $\mathrm{C}-\mathrm{O}$ and $\mathrm{C}=\mathrm{O}$ bonds of CUNaT were recognized by the XRD and FTIR studies. Novelty: The monoclinic structure and space group $\mathrm{P} 2{ }_{1}$ of grown CuNaT crystal was non-identical to the orthorhombic structure of the copper tartrate crystal was enabled to identify its novel structure. The NLO competence of the CUNaT was 0.38 times relative to KDP and of higher LDT value than the KDP, provided its novel optical properties. Applications: The strong intensity emission peaks recorded at $297 \mathrm{~nm}$ and $380 \mathrm{~nm}$ in the photoluminescence spectrum and the lower absorption edge at $334 \mathrm{~nm}$ in the UV spectrum of the CuNaT crystal exhibited its potential use in non-linear optics.

Keywords: Single crystal XRD; FTIR; UVVisNIR; PL; SHG; LDT

\section{Introduction}

Organometallic materials have a great impact on information technology and nonlinear optical applications ${ }^{(1)}$. In general, the metal organic polymers or coordination polymers as non-linear optical materials are that molecular architectures can be easily modulated to optimize the microscopic factors, such as polarization, ionic conductivity, and the related macroscopic parameters such as electrical conductivity ${ }^{(2)}$ and antimicrobial activity ${ }^{(3)}$.Consideration of these properties, nowadays coordination polymers with an excellent optical non-linearity have been the focus on current research activity by knowing their potential applications in optical and biological fields. To accomplish this criterion, tartaric acid derived metal complexes are chosen with their 
ionically bonded metal ions in the metal-tartrate structure matrix, form donor ligand of chiral based three dimensional molecular networks ${ }^{(4)}$.

Especially metal tartrate complexes earn individual attention for the reason of their several remarkable structural ${ }^{(5)}$, spectral, non-linear optical, thermal ${ }^{(6)}$ and biological ${ }^{(7)}$ properties. The tartaric acid is combined with the divalent $\mathrm{Cu}$ (II) metal ions to form a metal tartrate crystalline complex which mainly contains $\mathrm{O}-\mathrm{H}, \mathrm{C}-\mathrm{H}, \mathrm{C}-\mathrm{H}-\mathrm{O}$ and $\mathrm{O}-\mathrm{H}-\mathrm{O}$ bonds ${ }^{(8)}$. These hydrogen bonds and the ionic bonds between metal ions of metal tartrate crystalline complex are the fundamental reason for their remarkable non-linear optical properties. Further, the structural flexibility of metal tartrates are simply adaptable through an exact chemical reaction for increasing the chirality centers in order to obtain the requirements of non-centro symmetry, surface quality and non-linear optical activity based materials to utilize their applications in the fields of non-linear optics ${ }^{(9)}$. Mostly, pure metal tartrate material has sparingly solubility character in water and it decomposes quickly before attaining its melting point. Thus, the gel process provides an effective advantage to grow material designed for better crystallization. Recently, the growth, vibrational and optical properties of the silica gel grown bimetallic potassium strontium tartrate and potassium calcium tartrate have been published ${ }^{(10)}$. Further, efforts have been built to learn the LDT effect on the tartaric acid single crystal reported already that the LDT value of the pure tartaric acid crystal was less than the doped tartaric acid crystal ${ }^{(11)}$. While we find an adequate literature survey on optical, thermal and dielectric ${ }^{(12)}$ in mixture of metal tartrates using gel technique, there is very less information about the linear and non-linear characteristic properties of copper sodium tartrate, and this present paper intends to account its optical properties for finding applications in non- linear optical fields.

Hence, in the present investigation, we proposed to study about the linear and non-linear optical properties of the copper sodium tartrate single crystal using the single crystal XRD, UV-vis-NIR, photoluminescence, laser damage threshold, and SHG characterizations for finding its applications in the field of non-linear optics.

\section{Materials and Method}

\subsection{Materials}

The copper sodium tartrate single crystal was grown in silica gel medium by single-diffusion chemical reaction method. The highly AR grade purified materials purchased from LOBA Chemie goods of cupric chloride (99.9\% assay), sodium meta silicate (99.9\% assay), sodium chloride (99.9\% assay) and tartaric acid (99.9\% assay). They were used for starting materials to grow the copper sodium tartrate crystal.

\subsection{Synthesis and growth of $\left[\mathrm{Na}_{2} \mathrm{Cu}_{2}\left(\mathrm{C}_{4} \mathrm{H}_{6} \mathrm{O}_{6}\right)_{3}\left(\mathrm{H}_{2} \mathrm{O}\right)_{3}\right] 3 \mathrm{H}_{2} \mathrm{O}$ crystal}

The crystallizer consists of a glass tube of length $100 \mathrm{~mm}$ and diameter $25 \mathrm{~mm}$. Sodium meta silicate of specific gravity $1.04 \mathrm{~g} / \mathrm{cc}$ was mixed with Tartaric acid (2M) to obtain hydro silica gelled into $\mathrm{pH}$. The gel of the given pH 5 was then permitted to set in the given time from 10 hours to 40 hours. Once the gel got set, it was shifted to different test tubes; half filled of its volume and then kept undisturbed in 72 hours of proper dilution. When sodium metasilicate reacts with the solvent, the expected products of sodium hydroxide and silisic acid are produced. An aqueous solution to $2 \mathrm{M}$ cupric chloride was added to the set gel slowly along the walls of the tube to avoid gel breakage. The tube was kept undisturbed at room temperature.

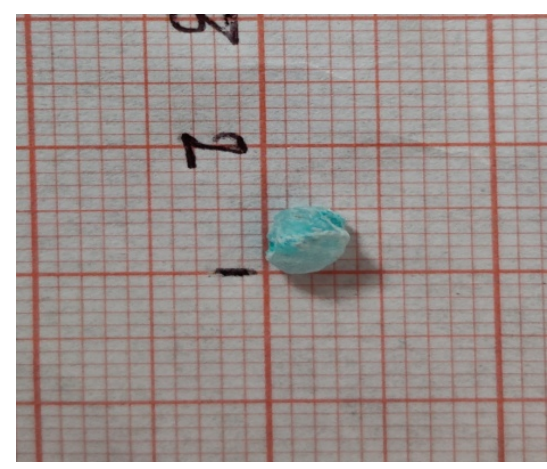

Fig 1. Size of copper sodium tartrate single crystal 
The tartaric acid was reacted with sodium hydroxide and the cupric chloride, creating the sodium copper tartrate crystals through the reaction,

$$
\begin{gathered}
2 \mathrm{Na}_{2} \mathrm{SiO}_{3}+6 \mathrm{H}_{2} \mathrm{O} \rightarrow 2 \mathrm{H}_{4} \mathrm{SiO}_{4}+4 \mathrm{NaOH} \\
4 \mathrm{NaOH}+3 \mathrm{C}_{4} \mathrm{H}_{6} \mathrm{O}_{6}+2 \mathrm{CuCl}_{2} \mathrm{O} .5 \mathrm{H}_{2} \mathrm{O} \rightarrow\left[\mathrm{Na}_{2} \mathrm{Cu}_{2}\left(\mathrm{C}_{4} \mathrm{H}_{4} \mathrm{O}_{6}\right)_{3}\left(\mathrm{H}_{2} \mathrm{O}\right)_{3}\right]+2 \mathrm{NaCl}+3 \mathrm{H}_{2} \mathrm{O}
\end{gathered}
$$

The newly grown $\left[\mathrm{Na}_{2} \mathrm{Cu}_{2}\left(\mathrm{C}_{4} \mathrm{H}_{4} \mathrm{O}_{6}\right)_{3}\left(\mathrm{H}_{2} \mathrm{O}\right)_{3}\right] 3 \mathrm{H}_{2} \mathrm{O}$ single crystals [Figure 1] were kept without disturbance for one week in the test-tube in order to have large size single crystals for the non-linear optical characterization analyses.

\subsection{Characterization Methods}

Single crystal X-ray diffraction analysis was done by using the single crystal ENRAF NONIUS CAD4 X-Ray diffractometer equipped with $\operatorname{MoK} \alpha\left(\lambda=0.71073 \mathrm{~A}^{\circ}\right)$ radiation was employed to estimate the unit cell parameters, crystal system and space group. The grown crystals of UV-VIS-NIR spectra were recorded from $190 \mathrm{~nm}$ to $1100 \mathrm{~nm}$ wavelength region by Lambda 35 spectrometer. Photoluminescence properties were investigated in solutions at room temperature by Cary Eclipse Spectro Fluro meter in the range from $240-400 \mathrm{~nm}$ with the excitation wavelength of $275 \mathrm{~nm}$. The second order non-linear optical efficiency of the as-grown CuNaT crystal is calculated by Kurtz-Perry powder technique. For the present sample, the KDP crystal was used as the standard reference material. The Laser Damage Threshold of as-grown CuNaT crystal was calculated by a Q-switched pulsed Nd: YAG $(1064 \mathrm{~nm})$ laser. The diameter and pulse width of a laser beam are $1 \mathrm{~m}$ and 10 ns was used to irradiate on the crystal surface in the frequency rate of $10 \mathrm{~Hz}$.

\section{Results and Discussion}

\subsection{Single crystal X-ray diffraction analysis}

Crystal structure of $\left[\mathrm{Na}_{2} \mathrm{Cu}_{2}\left(\mathrm{C}_{4} \mathrm{H}_{4} \mathrm{O}_{6}\right)_{3}\left(\mathrm{H}_{2} \mathrm{O}\right)_{3}\right]_{n} 3 \mathrm{H}_{2} \mathrm{O}$ was solved [Table 1] by single crystal x-ray diffraction analysis and found that the structure of the CuNaT crystal belongs to monoclinic with P2 1 space group. The CCDC number 1964952 was assigned for the as-grown crystal. The slightly different change in the unit cell parameters than the copper tartrate ${ }^{(11)}$ proved the lattice tension on sodium metal toughened owing to inclusion of copper tartrate crystal.

Table 1. Calculated lattice factors of CuNaT Crystal

\begin{tabular}{ll}
\hline Copper sodium tartrate & Calculated values of Lattice parameters \\
\hline Molecular formula & {$\left[\mathrm{Na}_{2}\left(\mathrm{Cu} 2\left(\mathrm{C}_{4} \mathrm{H}_{4} \mathrm{O}_{6}\right)_{3}\left(\mathrm{H}_{2} \mathrm{O}\right)_{3}\right)\right]_{n} 3 \mathrm{H}_{2} \mathrm{O}$} \\
Molecular weight & 725.37 \\
Structural parameters & $a=7.7230(5): \alpha=90^{\circ}:$ \\
& $\mathrm{b}=20.2964(11): \beta=113.675(2)^{\circ}:$ \\
& $c=7.9896(5): \gamma=90^{\circ}$ \\
Crystal size & $(0.150 \times 0.150 \times 0.100) \mathrm{mm} 3$ \\
Volume & $1146.96(12) \AA 3$ \\
Theta range for data collection & $2.784^{\circ}$ to $32.955^{\circ}$ \\
Index ranges Density & $11<=\mathrm{h}<=11,-30<=\mathrm{k}<=29,-12<=\mathrm{l}<=11 \mathrm{Z}=2$ \\
\hline
\end{tabular}

In the copper sodium tartrate crystal structure, the positively charged sodium $(+)$ and copper $(2+)$ ions were influenced by the negatively charged tartrate group known as the carboxylate group. During crystallization, the process of proton transport mechanism through carboxylate groups indicated the formation of a crystal.

In the tartrate group of CuNaT crystal, the hydroxyl and carboxyl oxygen atoms contributed in different $\mathrm{C}$-H...O and $\mathrm{O}$ $\mathrm{H}$... O exchange interactions for increasing its optical properties and participated a sole role for creating its crystalline structure. The carbon frame from the tartrate group was basically planar [torsion angle C9-C10-C11-C12 of $170.1^{\circ}(2)$ ] and the direction of angle among the hydroxyl carboxyl assembly $\left[\mathrm{O}(3)-\mathrm{C}(2)-\mathrm{H}(2)\right.$ was $123.4^{\circ}$ (3) and $\mathrm{O}(2)-\mathrm{C}(1)-\mathrm{O}(1)$ was $\left.107.9^{\circ}\right]$ were high compared with the tartaric acid ${ }^{(13)}$. The different in variation of C-H...O and O-H..O $\left[\mathrm{H}(23 \mathrm{~A})-\mathrm{O}(23)-\mathrm{H}(23 \mathrm{~B})\right.$ was $99^{\circ}(3)$ and $\mathrm{H}(24 \mathrm{~A})-\mathrm{O}(24)-\mathrm{H}(24 \mathrm{~B})$ was $112^{\circ}(3)$ ] bond angles illustrated electronic vibrations of CuNaT crystal exploring about its non-linear optical properties. 


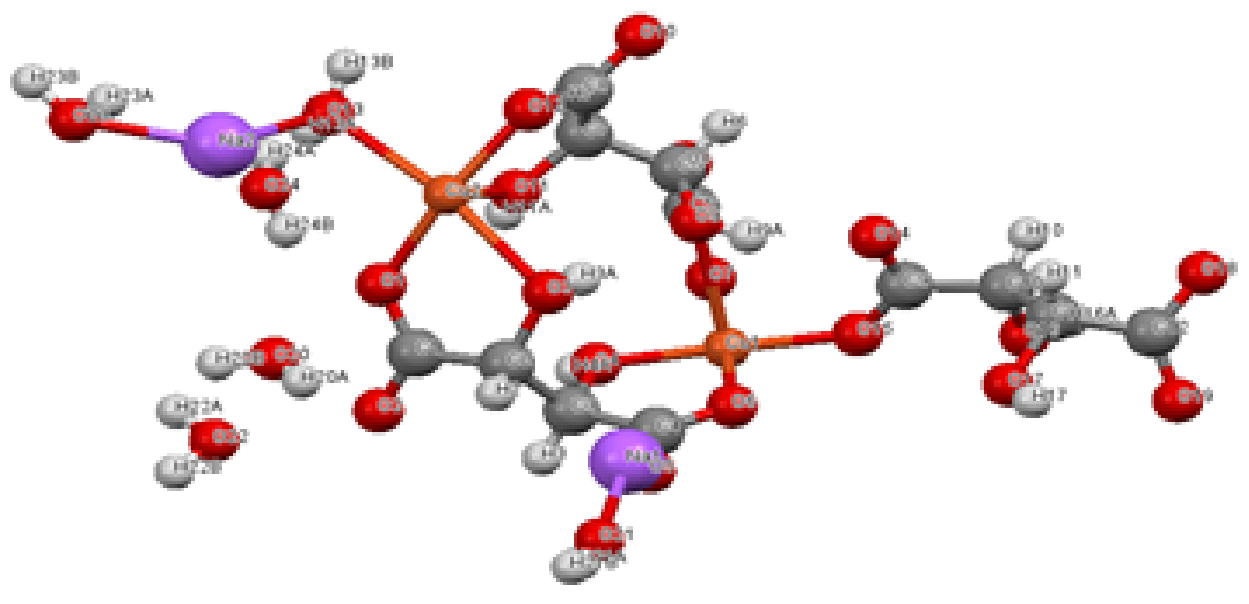

Fig 2. Atom numbering structure of CuNaT crystal

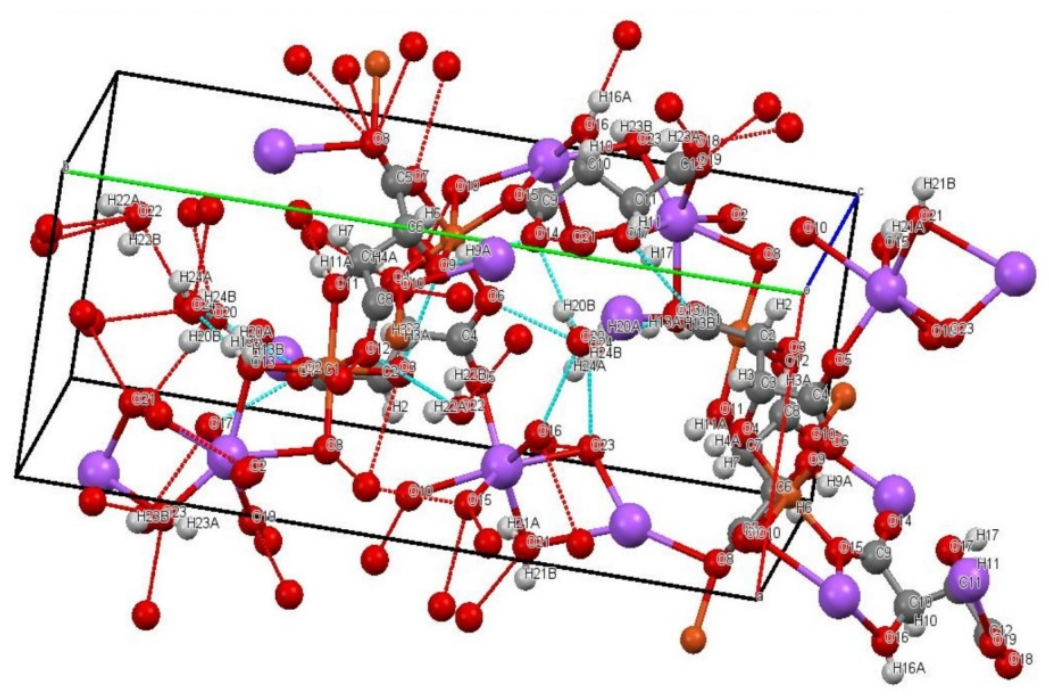

Fig 3. Hydrogen bonds structure of CuNaT crystal

The inter- and intra-molecular hydrogen-bonding interactions intended to give the 3D molecular structure [Figure 3] of the $\mathrm{NaCuT}$ crystal along b-axis contains both coordinated and solvated water molecules.

\subsection{Optical absorption analysis}

UV-vis-NIR spectrum of copper sodium tartrate crystal of thickness $1.5 \times 10^{-3} \mathrm{~m}$ was plotted from 200 to $800 \mathrm{~nm}{ }^{(14)}$ of the wavelength range covering the whole ultraviolet region. The absorption peaks for CuNaT crystal with cut-off wavelengths at $334 \mathrm{~nm}$ in the UV region may be assigned to $80 \%$ good transmittance in the visible region. Similar absorption established at $328 \mathrm{~nm}$ for bimetallic potassium strontium tartrate crystal has been reported ${ }^{(15)}$. The lower cutoff wavelength of the present $\mathrm{CuNaT}$ crystal is attributed to the resonance assisted bands from the carboxylate group $\left(\mathrm{COO}^{-}\right)$of $\mathrm{CuNaT}$ to metal $\left(\pi\right.$ to $\left.\pi^{*}\right)$ transitions ${ }^{(16)}$. 


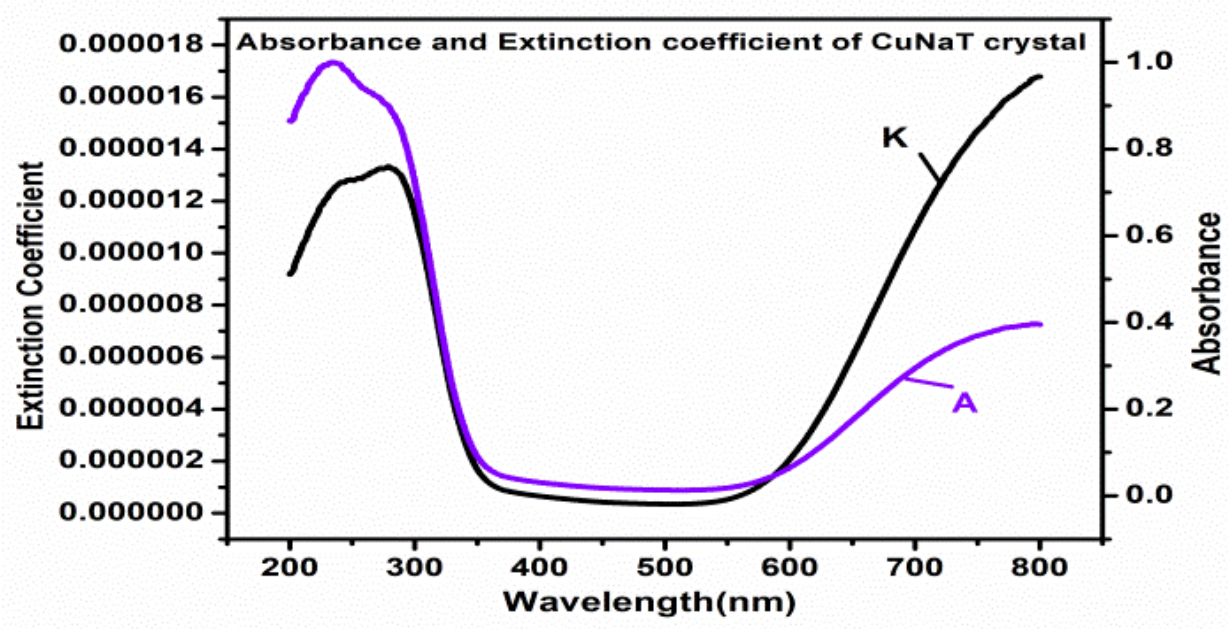

Fig 4. UV-vis-NIR absorbance and extinction coefficient vs. wavelength plots of CuNaT crystal

The functional electromagnetic light wavelength changes with the extinction coefficient of grown copper sodium tartrate crystal and is shown in [Figure 4]. The reduced value of extinction coefficient from $350 \mathrm{~nm}$ to $550 \mathrm{~nm}$ specified that the loss of incident electromagnetic radiation was low as the scattering was very less. Simultaneously, the increased value of extinction coefficient beyond $550 \mathrm{~nm}$ confirmed that the higher loss of electromagnetic radiation was due to the absorption of incident photons and the scattering centres of as grown CuNaT crystal.

The expression used to calculate the linear optical absorption coefficient is given by the expression,

$$
\alpha=\frac{2.303}{t} \log \frac{1}{T}
$$

Where $\mathrm{T}$ stands for the transmittance and $\mathrm{t}$ corresponds to the thickness of the copper sodium tartrate crystal. The optical absorption coefficient was the genuine factor for finding the penetrating power of the incident light ray. If the absorption coefficient at $334 \mathrm{~nm}$ [Figure 5] was (139.84) low, the incident light was poorly absorbed by the present sample. Moreover if that absorption coefficient was high at the cut-off edge, it was highly absorbed by the material and expressed the elevated optical trouncing property of the grown CuNaT crystal.

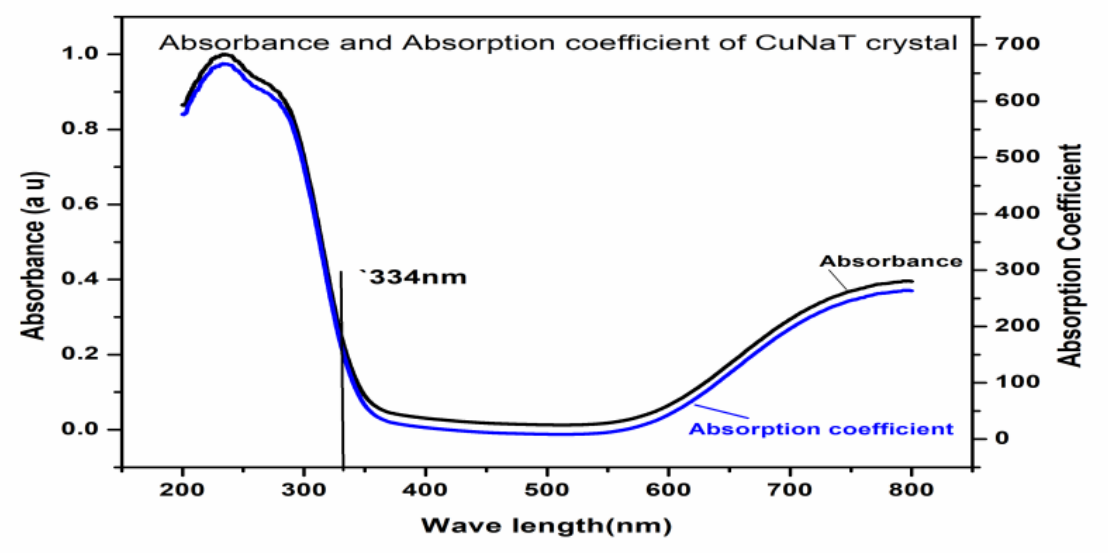

Fig 5. UV-vis-NIR Absorbance and Absorption coefficient vs. wavelength plots of CuNaT crystal 
The optical parameter $\alpha$ proposed a direct band gap ${ }^{(17)}$ of the as-grown crystal using the relation,

$$
(\alpha h \gamma)^{2}=A\left(E_{g}-h \vartheta\right)
$$

Where, h represents the Plank's constant, A is an Absorbance, $v$ stands for the high energy incident photon's frequency and Eg corresponds to the band gap.

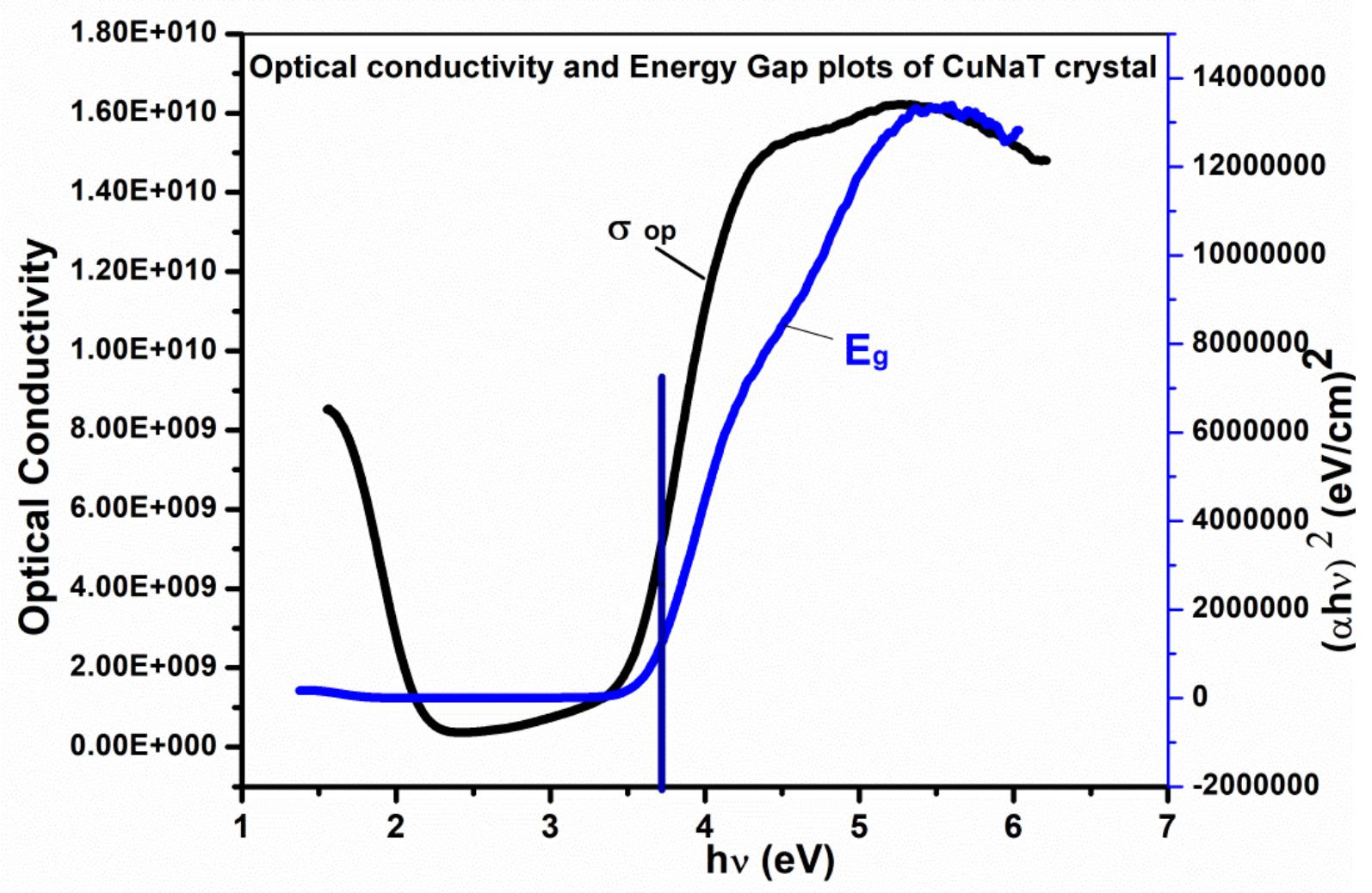

Fig 6. UV-vis-NIR optical conductivity and Energy gap vs. Photon energy plots of CuNaT crystal

The calculated direct band gap energy of the as-grown crystal was computed to be $3.71 \mathrm{eV}$ using the given relation,

$$
E_{g}=\frac{1240}{\lambda} \mathrm{eV}
$$

where $\lambda$ represents the lower edge absorption wavelength $(334 \mathrm{~nm})$. The optical conductivity is determined at $\lambda=334 \mathrm{~nm}$ using the relation,

$$
\sigma_{o p}=\frac{\alpha n c}{4 \pi}
$$

The optical conductivity is one of the optical parameters to study the nature of the electronic states in a grown material. The [Figure 6] shows that the above the incident photon energy $3.71 \mathrm{eV}$, the optical conductivity increases with the increase of photon energy ${ }^{(18)}$. This estimated energy value exposed the semiconducting nature of the CuNaT crystal finding its usefulness for manufacturing diodes in electronics. 


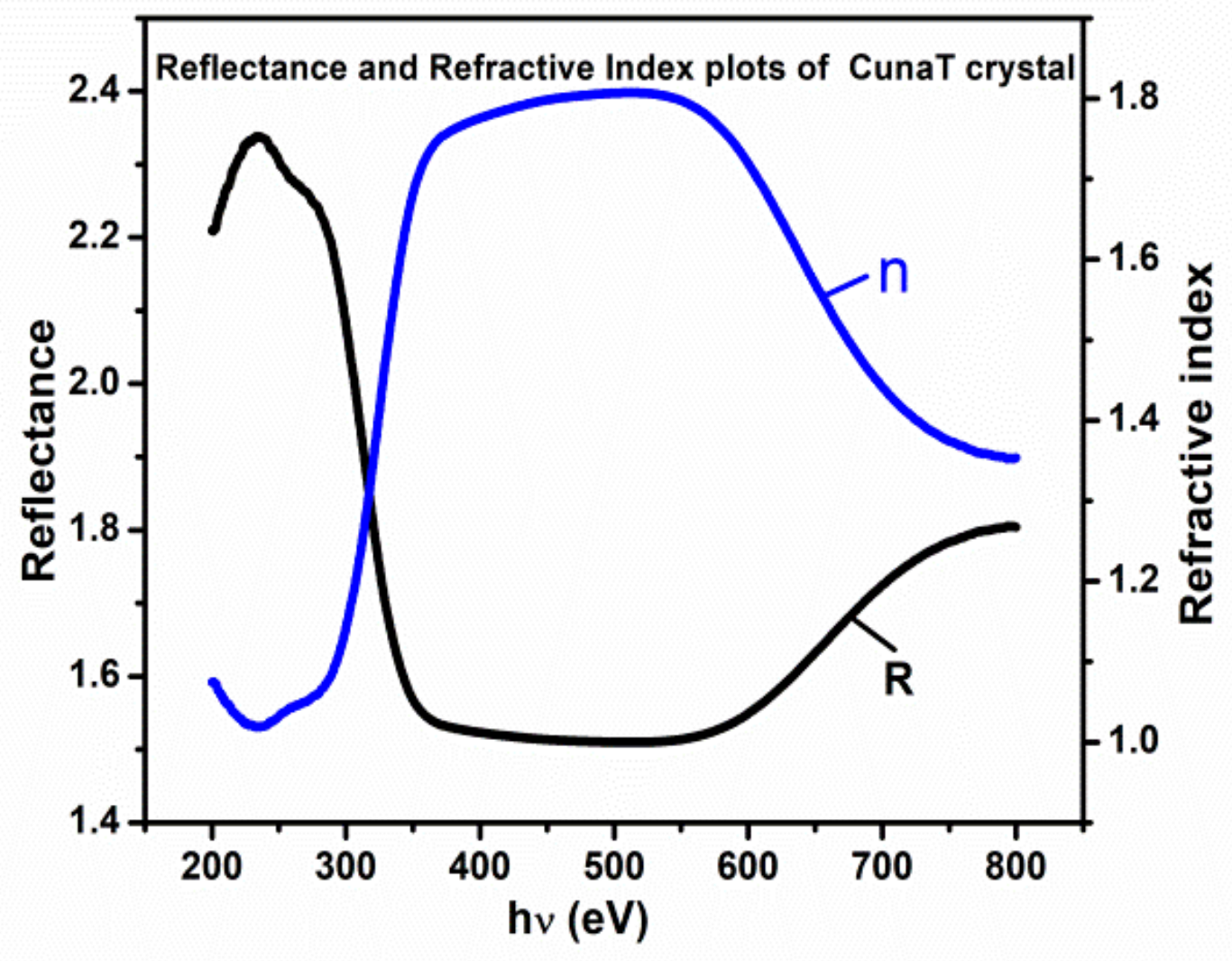

Fig 7. Refractive index and Reflectance vs. Photon Energy of CuNaT crystal

Reflectance is one among the good optical parameter which is determined at $\lambda=334 \mathrm{~nm}$ in terms of the absorption coefficient relation,

$$
R=1 \pm \frac{\sqrt{(1-\exp (-\alpha t)+\exp (\alpha t))}}{1+\exp (-\alpha t)}
$$

The relation which is used to find the refractive index of as grown crystal at $\lambda=334 \mathrm{~nm}$ is by applying reflectance,

$$
n=-\frac{\left((R+1) \pm \sqrt{\left.\left(3 R^{2}+10 R-3\right)\right)}\right.}{2(R-1)}
$$

The constant fall in the optical parameters of reflectance and refractive index favouring the Photon energy of copper sodium tartrate crystal are shown in [Figure 7]. When the Photon energy increased, the reflectance and refractive index of as-grown crystal decreased which identified that the incident photons interacted with the electrons of the grown CuNaT crystal exhibiting charge transformations ${ }^{(19)}$. In addition, the low values of reflectance and refractive index at the first absorption edge was mainly done by the metal to ligand transformation of as-grown crystal. Thus, the initial absorption edge revealed the $n$ to $\pi^{\star}$ charge mobilization mechanism of electrons from the carboxylic group of CuNaT crystal. Further, the lower edge optical absorption in the UV area of the CuNaT spectrum revealed the defect free and purity nature of the present sample for higher order optical characterizations. 


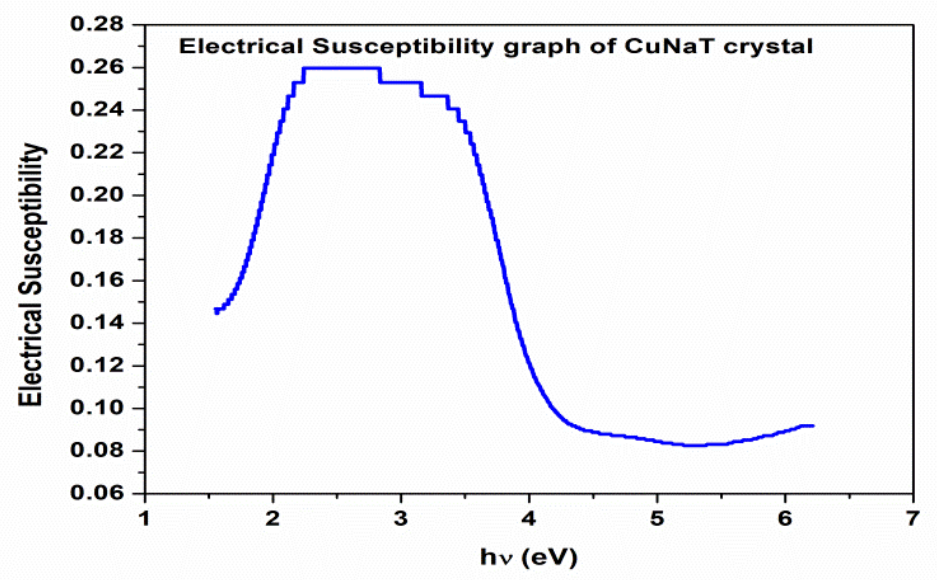

Fig 8. Variation of electrical susceptibility with incident photon energy of CuNaT crystal

The calculated electrical susceptibility $\left(\chi_{c}\right)$ value ${ }^{(20)}$ at $\lambda=334 \mathrm{~nm}$ using the parameters of extinction coefficient and refractive index by the relation,

$$
\begin{gathered}
\varepsilon_{r}=\varepsilon_{0}+4 \pi \chi=n^{2}-k^{2} \\
\chi=\frac{n^{2}-k^{2}-\varepsilon_{0}}{4 \pi}
\end{gathered}
$$

From the [Figure 8], the electrical susceptibility is decreased for increasing the incident photon energy of as grown CuNaT crystal lead to the change in electronic band structure.

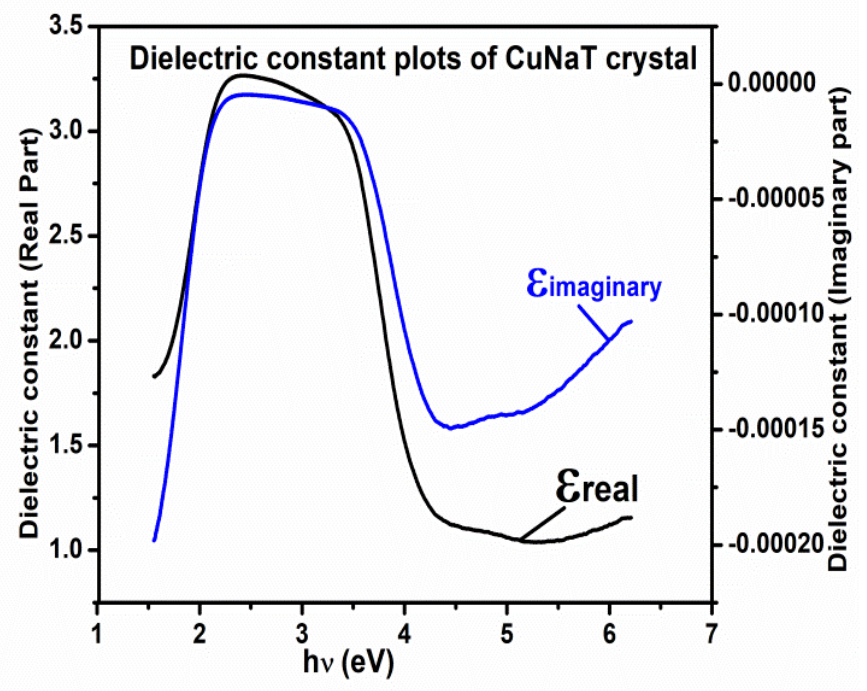

Fig 9. Variation of dielectric constant imaginary part and Dielectric constant real part with incident photon energy of CuNaT crystal 
Dielectric constant was calculated at $\lambda=334 \mathrm{~nm}$ from the expressions given below ${ }^{(21)}$,

$$
\begin{gathered}
\in=\varepsilon_{r}+i \varepsilon_{i}=(n+i k)^{2} \\
\varepsilon_{r}+i \varepsilon_{i}=n^{2}-k^{2}+2 n i k
\end{gathered}
$$

where $\varepsilon_{r}$ corresponds to the real part of dielectric constant and was calculated by the relation,

$$
\begin{gathered}
\varepsilon_{r}=n^{2}-k^{2} \\
\varepsilon_{i}=2 n i k
\end{gathered}
$$

The [Figure 9] showed that the real part of the dielectric constant was decreased more than the imaginary part for the applied incident photon energy. A superior value of dielectric constant in lesser frequencies are by the lesser electrostatic binding force

\begin{tabular}{|c|c|c|c|c|c|c|}
\hline \multirow{2}{*}{$\begin{array}{l}\text { Extinction } \\
\text { coefficient ( } \mathrm{k})\end{array}$} & \multirow{2}{*}{$\begin{array}{l}\text { Reflectance } \\
\text { (R) }\end{array}$} & \multirow{2}{*}{$\begin{array}{l}\text { Refractive Index } \\
\text { (n) }\end{array}$} & \multirow{2}{*}{$\begin{array}{l}\text { Electrical } \\
\text { susceptibility }\left(\chi_{c}\right)\end{array}$} & \multirow{2}{*}{$\begin{array}{l}\text { Optical } \\
\text { conductivity } \sigma_{(p)}\end{array}$} & \multicolumn{2}{|c|}{ Dielectric constant $(\varepsilon)$} \\
\hline & & & & & Real $\varepsilon r$ & $\operatorname{Im} \varepsilon i$ \\
\hline $3.71 \mathrm{E}-06$ & 1.66 & 1.53 & 0.18 & $5.11 \mathrm{E}+009$ & 2.33 & $-4.6 \mathrm{E}-05$ \\
\hline
\end{tabular}
that arises through space charge polarization around the granule edge. At elevated frequencies, dipoles are not able to track applied frequencies. Therefore, a decrement in polarizations shrinks the values of dielectric constant in high frequencies ${ }^{(22)}$.

Table 2. Calculated optical factors of copper sodium tartrate crystal

The measured optical factors of CuNaT crystal at its energy gap 3.71eV were given in Table 2. The calculated optical constants confirmed that the CuNaT is a competent material in the emerging laser and electronics fields.

\subsection{PL studies}

The photoluminescence spectrum of copper sodium tart rate crystal was recorded using Cary Eclipse Spectro Fluro meter in the range $400 \mathrm{~nm}-700 \mathrm{~nm}$ for the excitation wavelength of $420 \mathrm{~nm}$. The excited emission peaks of the as-grown crystal observed at $598.5 \mathrm{~nm}$, and $635.8 \mathrm{~nm}$ illustrated the less intrinsic defects of the CuNaT crystal ${ }^{(23)}$.

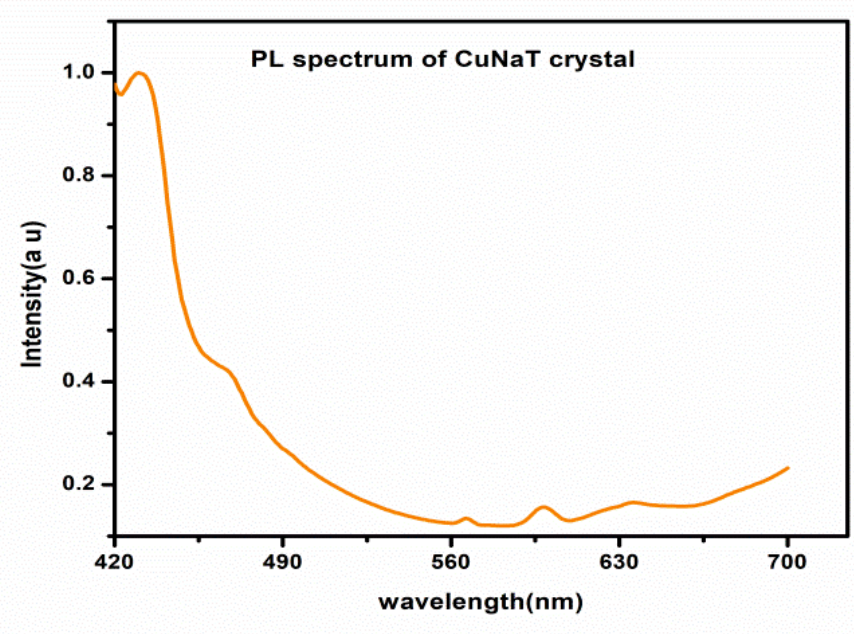

Fig 10. Photo luminescence excitation peaks of copper sodium tartrate crystal.

The observed peaks [Figure 10] of orange light emission revealed that it was a budding material for fabricating resonators and light emitting diodes in the opto- electronics industries. 


\subsection{SHG analysis}

The as-grown CuNaT crystal is obtained in the powdered form after crushing well by using the mortar and pestle. The nonlinear optical property of the CuNaT crystal was analyzed by using Q Switched Nd: YAG laser having wavelength of $1064 \mathrm{~nm}$ with input beam energy of $450 \mathrm{~mJ}$ and pulse width of $10 \mathrm{~ns}$ with repetition rate of $10 \mathrm{~Hz}$. For interacting well with the laser light, the powdered form of the as - grown crystal is made as pellets by using the pelletizer. The microcrystalline fine powder models of various sizes ( 25 to $300 \mu \mathrm{m}$ ) were packed in capillary tubes and lighted by this laser. From 25 to $100 \mu \mathrm{m}$ the effectiveness of green emission enhances, after attaining a saturation rate, there is no longer rise with the rise of particle size which revealed the phase matching capability of the CuNaT crystal. With an input rate of $10 \mathrm{~Hz}$, one half of the splitted laser beam was passed to the sample by the centre of the concave mirror and the other half was sent to the photodiode for calculation. The output of the CuNaT crystal was calculated as $58 \mathrm{mV}$ for input beam energy of $5 \mathrm{~mJ} /$ Pulse at the $95 \mu \mathrm{m}$ of particle size. Whereas; the $\mathrm{KDP}$ crystal provided an output of $156 \mathrm{mV}$ for the similar input signal. The green light emission $(\mathrm{l}=532 \mathrm{~nm})$ from the CuNaT crystal proved that its novelty of non centrosymmetric crystal structure for confirming its nlo activities. The SHG efficiency of L- Prolinium Tartaric acid ${ }^{(24)}$ and p-Toluidinium L-Tartrate ${ }^{(25)}$ have been reported and found that the values were marginally varied with the present CuNaT crystal.

\subsection{Laser-induced damage threshold analysis}

The surface quality of the as-grown crystal depends on the ability to withstand the high power laser intensities leading to identify its application in the non-linear optical fields. The crystal was placed at the focus of the $35 \mathrm{~cm}$ focal length of converging lens where the incident laser beam was controlled. During the irradiation, a power meter measured the energy density $\left(\mathrm{P}_{d}\right)$ of the input laser beam at the point of damage to the crystal using the relation,

$$
P_{d}=\frac{E}{\tau \pi r}
$$

Where $\mathrm{E}$ is the input energy density (mJ), $\tau$ is the pulse width (ns), and $\mathrm{r}$ is the area of the circular spot $(\mathrm{mm})$. The calculated good laser damage threshold value of $\mathrm{NaCuT}$ crystal was found to be $1.4 \mathrm{GW} / \mathrm{cm}^{2}$ which is higher than the LDT values of $\operatorname{KDP}(0.20)^{(26)}$, Ammonium hydrogen L-tartrate ${ }^{(27)}$ and 8-hydroxyquinonium hydrogen maleate ${ }^{(28)}$.

\section{Conclusion}

The new organometallic copper sodium tartrate single crystal was grown by silica gel medium. The newly solved monoclinic structure, non -centrosymmetry with $\mathrm{P} 21$ space system of the as-grown copper sodium tartrate single crystal favouring as novel structure was confirmed by single crystal XRD. From the UV spectral studies, the calculated higher value of the band gap energy $(3.7 \mathrm{eV})$ combined with the lower cut-off wave length at $334 \mathrm{~nm}$ and good optical transmittance found the appropriateness of the present material being used for preparing optical filters and semiconducting diodes. The high intensity orange light emission peaks at $566.45 \mathrm{~nm}$ and $598.5 \mathrm{~nm}$ in visible region of PL spectrum revealed the good crystalline nature of as grown CuNaT crystal. The variation of incident photon energy with electrical susceptibility, optical conductivity, and dielectric constant illustrated that its polar nature, transformations of charge carriers and dipole behavior of a grown sample found its usefulness for fabricating spectral and dispersion devices in the field of optical and electrical fields. The non centrosymmetry structure of grown CuNaT crystal was confirmed through its green light emission ( $\mathrm{I}=532 \mathrm{~nm}$ ) from SHG Analysis. Further, the NLO efficiency of the CuNaT crystal was 0.38 times relative to KDP as shown by the SHG analysis. The high LDT value of the asgrown CuNaT crystal suggested that it found application in laser frequency conversion. From the current investigation, we bring to a conclusion that $\mathrm{CuNaT}$ is a good non-linear optical crystal for fabricating optical filters, nlo devices and electro plating materials in the electro-optical fields.

The future scope of the present study emphasized that although the gel growth method is preferred for getting a single crystals suitable for X-ray diffraction analysis rather than poly crystalline products if the products are poorly soluble, hydrothermal method is recommended to improve the size, purity with a high surface laser damage threshold (LDT) has become more of a demand in the frontier areas of optical switching and communications applications. By adding the combination of urea and Gum Arabic in mixed proportions led to the formation of nanocrystallites of metal dicarboxylates that are proved to be important in understanding the control of kidney stone formation in healthcare applications. 


\section{Acknowledgement}

Mrs. Daisy Selasteen acknowledges with thanks Mr. Singaraj, Rtd Vice Principal, Campion Anglo Indian Hr.Sec.School, Tiruchirappalli for his help and encouragement for preparing this manuscript.

\section{References}

1) Sheela GE, Manimaran D, Joe H, Jothy VB. Studies on Molecular Structure and Vibrational Spectra of NLO Crystal L-Glutamine Oxalate by DFT Method. Indian Journal of Science and Technology. 2017;10(31):1-23. Available from: https://dx.doi.org/10.17485/ijst/2017/v10i31/114486.

2) Greena JAM. Electrical conductivity studies on pure and barium added strontium tartrate trihydrate crystals. Indian Journal of Science and Technology. 2010;3(3):250-252. Available from: https://dx.doi.org/10.17485/ijst/2010/v3i3.22.

3) Revanasiddappa HD, Vijaya B, Shivakumar L, Prasad K, Revanasiddappa HD, Vijaya B, et al. Synthesis, Structural Characterization, and Antimicrobial Activity Evaluation of New Binuclear Niobium (V) Tartrate Complexes with Biologically Important Drugs. Inorganic Chemistry. 2013;p. 1-7. Available from: http://dx.doi.org/:10.1155/2013/760754.

4) Smith G, Wermuth UD. Three-dimensional hydrogen-bonded structures in the 1:1 proton-transfer compounds ofL-tartaric acid with the associativegroup monosubstituted pyridines 3-aminopyridine, 3-carboxypyridine (nicotinic acid) and 2-carboxypyridine (picolinic acid). Acta Crystallographica Section C Crystal Structure Communications. 2010;66(1):5-10. Available from: https://dx.doi.org/10.1107/s0108270109049154.

5) Bott RC, Sagatys DS, Lynch DE, Smith G, Kennard CHL. Structure of sodium hydrogen (+)-tartrate monohydrate. Acta Crystallographica Section C Crystal Structure Communications. 1993;49(6):1150-1152. Available from: https://dx.doi.org/10.1107/s0108270192012605.

6) Binitha MP, Pradyumnan PP. Thermal degradation, dielectric and magnetic studies on copper tartrate trihydrate crystals. Journal of Thermal Analysis and Calorimetry. 2013;114(2):665-669. Available from: https://dx.doi.org/10.1007/s10973-013-2998-2.

7) Patil CS, S DN, Pawar TVB, Synthesis R. Characterization of Novel Mixed Metal Tartrate Complexes and Study of their in vitro antimicrobial activity. Int J Pharm Sci Res. 2016;7(4):1524-1534. Available from: http://dx.doi.org/10.13040/IJPSR.0975-8232.

8) Al-Dajani TMM, Hassan H, Abdallah N, Mohamed. Madhukar Hema malini, Hoong-Kun Fun, Diaquabis (hydrogen tartrato) copper(II) dehydrate. Acta Crystallographica Section E: Crystallographic Communications. 2010;66:774-775. Available from: http://dx.doi.org/:10.1107/S160053681002115X.

9) Rajesh K, Kumar PP. Mechanical, thermal, linear and nonlinear optical properties of barium L-tartrate single crystal. Materials Research Express. 2017;4(1). Available from: https://dx.doi.org/10.1088/2053-1591/aa56cc.

10) Hemalatha K, Sumithra S, Gowri S, Kumar R. Influence of Metal on Vibrational and Optical Properties of Mixed Tartrate Crystal in Silica Gel Medium. Int J Cur Res Rev. 2017;9(10):133-136.

11) Jeyapappa K, Krishnan C, Selvarajan P. Growth, and studies of L-tartaric acid crystals doped with glycine. International Journal of Research and Analytical Reviews. 2018;5:847-855.

12) Joshi SJ, Tank KP, Vyas PM, Joshi MJ. Structural, FTIR, thermal and dielectric studies of gel grown manganese-copper mixed levo tartrate crystals. Journal of Crystal Growth. 2014;401:210-214. Available from: https://dx.doi.org/10.1016/j.jcrysgro.2014.01.060.

13) Jethva HO, Dabhi RM, Joshi MJ. Structural, Spectroscopic, Magnetic and Thermal Studies of Gel-Grown Copper Levo-Tartrate and Copper DextroTartrate Crystals. IOSR Journal of Applied Physics. 2016;8:33-42.

14) Zhu Z, Zhang Y, Ji Z, He S, Yang X. High-throughput DNA sequence data compression. Briefings in Bioinformatics. 2015;16:1-15. Available from: https://dx.doi.org/10.1093/bib/bbt087.

15) Hemalatha K, Sumithra S, Gowri S, Kumar R. Influence of Metal on Vibrational and Optical Properties of Mixed Tartrate Crystal in Silica Gel Medium. Int J Cur Res Rev. 2012;9:133-136.

16) Stanila A, Marcu A, Rusu D, Rusu M, David L. Spectroscopic studies of some copper(II) complexes with amino acids. Journal of Molecular Structure. 2007;834-836(836):364-368. Available from: https://dx.doi.org/10.1016/j.molstruc.2006.11.048.

17) Arora SK, Patel V, Chudasama B, Amin B. Single crystal growth and characterization of strontium tartrate. Journal of Crystal Growth. 2005;275(1-2):e657e661. Available from: https://dx.doi.org/10.1016/j.jcrysgro.2004.11.047.

18) Job CB, Shabu R, Paulraj S. Growth, structural, optical, and photo conductivity studies of potassium tetra fluoro antimonate crystal. Optik. 2016;127(8):3783-3787. Available from: https://dx.doi.org/10.1016/j.ijleo.2016.01.048.

19) Shankar G, Joseph PS, Suvakin MY, Sebastiyan A. Investigation of optical band gap in pyrrolidinomethylphthalimide crystal. Physica B: Condensed Matter. 2010;405(19):4231-4234. Available from: https://dx.doi.org/10.1016/j.physb.2010.07.016.

20) Dillip GR, Bhagavannarayana G, Raghavaiah P, Raju BDP. Effect of magnesium chloride on growth, crystalline perfection, structural, optical, thermal and NLO behavior of $\gamma$-glycine crystals. Materials Chemistry and Physics. 2012;134(1):371-376. Available from: https://dx.doi.org/10.1016/j.matchemphys. 2012.03.004.

21) Wankhade PM, Gambhire AB, Muley GG. Influence of urea doping on optical, thermal, mechanical and electrical properties of l-arginine phosphate monohydrate crystals for NLO applications. Optik. 2016;127(6):3322-3328. Available from: https://dx.doi.org/10.1016/j.ijleo.2015.12.091.

22) Gunasekaran S, Anand G, Balaji RA, Dhanalakshmi J, Kumaresan S. Crystal growth and comparison of vibrational and thermal properties of semi-organic nonlinear optical materials. Pramana. 2010;75(4):683-690. Available from: https://dx.doi.org/10.1007/s12043-010-0148-y.

23) Sawant DK, Patil HM, Bhavsar DS, Patil JH, Girase KD. Structural and Optical Properties of Calcium Cadmium Tartrate. Archives of Physics Research. 2011;2(2):67-73. Available from: http://scholarsresearchlibrary.com/archive.html.

24) Renuka K, Row TG, Prasad BR, Subramanian CK, Bhattacharya S. Hydrogen- bond- directed nonlinear-optical organic materials-evidence for the control of second harmonic generation activities from the X-Ray crystal structures of the complexes of L-Tartaric acid with M-Anisidine and P-Toluidine. New J Chem. 1995;19:83-89. Available from: http://eprints.iisc.ac.in/id/eprint/38026.

25) Suresh S, Devi SR, Sornamurthy BM, Arivanandhan M, Kumar RM. Growth, structural and optical studies of a novel nonlinear optical material: pToluidinium L-Tartrate. Optik. 2019;185:651-656. Available from: https://dx.doi.org/10.1016/j.ijleo.2019.03.032.

26) Min OK. Rb2Na (NO3)3: A Congruently Melting UV-NLO Crystal with a Very Strong Second-Harmonic Generation Response. Crystals. 2016;6(42):1-14. Available from: http://dx.doi.org/10.3390/cryst6040042.

27) Hanumantharao R, Kalainathan S, Bhagavannarayana G, Madhusoodanan U. An extensive investigation on nucleation, growth parameters, crystalline perfection, spectroscopy, thermal, optical, microhardness, dielectric and SHG studies on potential NLO crystal - Ammonium Hydrogen 1-tartarte. Spectrochimica Acta Part A: Molecular and Biomolecular Spectroscopy. 2013;103:388-399. Available from: https://dx.doi.org/10.1016/j.saa.2012.10.044. 
28) Peramaiyan G, Pandi P, Vijayan N, Bhagavannarayana G, Kumar RM. Crystal growth, structural, thermal, optical and laser damage threshold studies of 8-hydroxyquinolinium hydrogen maleate single crystals. Journal of Crystal Growth. 2013;375:6-9. Available from: https://dx.doi.org/10.1016/j.jcrysgro. 2013.04.011. 